\title{
Chloride Resistance Behavior on Nano- Metaclayed Ultra-High Performance Concrete
}

\author{
Mohd Faizal Md Jaafar ${ }^{1,}$, Hamidah Mohd Saman ${ }^{2}$, Muhd Norhasri Muhd Sidek ${ }^{2}$, Noorli \\ Ismail $^{3}$, and Nur Farhayu Ariffin ${ }^{1}$ \\ ${ }^{1}$ Faculty of Civil Engineering and Earth Resources, Universiti Malaysia Pahang, 26600 Gambang, \\ Pahang, Malaysia \\ ${ }^{2}$ Faculty of Civil Engineering, Universiti Teknologi MARA, 40450 Shah Alam, Selangor, Malaysia \\ ${ }^{3}$ Faculty of Civil and Environmental Engineering, Universiti Tun Hussein Onn Malaysia, 86400 Parit \\ Raja, Johor, Malaysia
}

\begin{abstract}
Nowadays, the application of nano materials is getting attention to enhance the conventional concrete properties. The ultrafine particles of nano material also will help reducing the formation of micro pores by acting as a filler agent, produce a very dense concrete and will reduce the growth of micro pores in the UHPC structures. The introduction of nano materials in concrete is to increase the strength and durability of concrete. One of the most important factor affecting concrete durability is chloride penetrability. The purpose of this study was to evaluate the chloride permeability and chloride penetration depth by using rapid chloride permeability test (RCPT) and colorimetric method, respectively. The study was conducted for normal performance concrete (NPC), high performance concrete (HPC), ultra-high performance concrete (UHPC) and a series of UHPC (nano metaclayed-UHPC) incorporating different replacement levels of nano metaclay. All the tests were performed at ages of concretes from 3 up to 365 days. The results showed that the presence of silica fume in HPC and nano metaclay in UHPC reducing the Coulombs passed on the 56-days up to 365-days. The experimental results also revealed the depth of chloride ion penetration for nano metaclayed-UHPC concretes are much lesser than those concretes. As regards to the results, nano metaclay led to noticeable benefit in term of chloride resistance.
\end{abstract}

\section{Introduction}

The most important characteristic of concrete is believed to be the one that affecting its durability of concrete. Nowadays, concrete with excellent performance in strength has become one of the most important construction materials. Conversely, it can be misleading to spontaneously assume that such concrete would also possess high performance qualities in marine environment [1]. A lot of deterioration is reported for marine construction since marine environments are very aggressive as well as sea water consists mainly of chlorides and sulphates [2]. As well-known, the major cause for degradation of reinforced concrete

\footnotetext{
* Corresponding author: faizaljaafar@ump.edu.my
} 
(RC) due to chloride penetration has been the great research effort until today. RC is usually durable, which has resulted in its widespread use for the construction of structures. However, it has become increasingly apparent that attack by aggressive agents such as chloride penetration can significantly affect a structure to deteriorate. Chloride ions can penetrate through the concrete pore network, potentially leading to the corrosion of steel reinforcement in the initiation stage [3]. The corrosion of reinforcing steel in concrete due to chloride penetration in RC structures has received increasing attention in recent years due to its widespread occurrence and the high cost of repair $[4,5]$.

It is important to note that the main feature of producing superior quality concrete is to minimize the volume of void in the concrete mass to have denser, stronger and more impermeable concrete. The interest in dealing with nano-cement and understanding of the hydration of cement particle to produce high strength concrete will be promised opportunity. Regards to this, the ultra-high performance concrete (UHPC) has been introduced as a new class and one of the most advanced concrete which it has better characteristics in term of strength and durability [6-8]. The use of industrial or agricultural by-product substitutions for cement has greatly contributed to boost up the performance characteristics of the concrete [9-11]. Therefore, the UHPC incorporating material as supplementary cementitious materials (CSM) and nano material has been developed. Previous literatures regard to the improved performance of UHPC containing pozzolanic cement enhanced the strength and increase the durability of concrete are well-documented in Schmidt et al. [12].

Numerous studies outlined that the use of nano-silica in UHPC can promote the hydration of cement to generate more $\mathrm{C}-\mathrm{S}-\mathrm{H}$ gel thus, leads to reduction of capillary pores in concrete [13-16]. Another researcher also found that UHPC contains ultra-fine POFA exhibited the greatest improvement in permeability properties such as porosity, rapid chloride permeability, water permeability and gas permeability [17]. In the same way, the chloride penetration in nanoclayed-UHPC were significantly improved its chloride resistance [18-20]. But, there are still few studies attempting to investigate chloride penetration in UHPC incorporating nano clay. The interest in using nano metaclay in UHPC production has been projected in this present research.

Regards to this, the ability of UHPC to resist chloride ion penetration is a key element for durability of concrete in salt-exposed environment was looked into. The rapid chloride permeability test (RCPT) results have been used as an indicator for concrete durability evaluation. However, several researchers have argued on this test when concrete produce with supplementary cementitious materials. For example, the used of silica fume in concrete mixture can lead to an order of magnitude reduction in $\mathrm{Na}+, \mathrm{K}+, \mathrm{Ca}+$ and $\mathrm{OH}-$ ion concentration in pore solution [21-23]. Therefore, it is not appropriate to use RCPT charge passed alone to evaluate chloride ions penetrability in concrete incorporating SCM [24].

However, Nokken and Hooton [25] argued with this outcome and they found the effect of using SCM in concrete is small. Therefore, additional method which is less dependent on the electrical conductance and pore solution is required to be validated. As a comparison with previous researches $[18,19]$, this study aimed to evaluated the chloride penetration profiles in various concrete types which are normal performance concrete (NPC), high performance concrete (HPC), ultra-high performance concrete (UHPC) and a series of UHPC integrating nano metaclay as cement replacement. For that reason, colorimetric method by using silver nitrate $\left(\mathrm{AgNO}_{3}\right)$ solution was performed other than RCPT. 


\section{Experimental program}

\subsection{Materials}

In this research, six (6) series of mixtures consists of normal performance concrete (NPC), high performance concrete (HPC), ultra-high performance concrete (UHPC) and UHPC with different percentage of nano metaclay (nano metaclayed-UHPC) were prepared. An ordinary Portland cement (OPC) Type I provided by local supplier was used as a binder for all mixes. The properties of OPC are equivalent to BS EN 197-1:2000 specification. The HPC mixture was prepared by replacing $10 \%$ silica fume (SF) from the total weight of OPC. Conversely, the nano metaclayed-UHPC mixture were formulated by replacing the OPC partly with different levels of nano metaclay at 1, 3 and $5 \%$ from the total weight of OPC used. The mixtures for nano metaclayed-UHPC were labelled as UHPC-1, UHPC-3 and UHPC-5 respectively. The preparation on calcination process; chemical composition and morphology examination on nano metaclay have been examined in previous studies [19]. The crushed gravel was used as the coarse aggregate with a nominal size of $10 \mathrm{~mm}$. Meanwhile, fine aggregate passing $5 \mathrm{~mm}$ was used as sand. In addition, the hypersuperplasticizier (SP) namely Glenium ACE389 SURTEC was added in NPC, HPC, UHPC and nano metaclayed-UHPC mixes to obtain a desired workability [20]. Table 1 displays the mix proportion of concrete for all mixes.

Table 1. Mix proportion for NPC, HPC, UHPC and nano metaclayed UHPC concretes

\begin{tabular}{lcccccccc}
\hline \multirow{2}{*}{$\begin{array}{c}\text { Concrete } \\
\text { Designation }\end{array}$} & OPC & SF & Metaclay & Aggregate & Sand & Water & SP & w/c \\
\hline NPC & 380 & - & - & 995 & 815 & 190 & 2.85 & 0.5 \\
HPC & 416 & 46 & - & 1149 & 619 & 152 & 4.20 & 0.4 \\
UHPC & 800 & - & 0 & 433 & 800 & 160 & 11.52 & 0.2 \\
UHPC-1 & 797 & - & 8 & 433 & 800 & 160 & 6.70 & 0.2 \\
UHPC-3 & 776 & - & 24 & 433 & 800 & 160 & 6.98 & 0.2 \\
UHPC-5 & 760 & - & 40 & 433 & 80 & 160 & 7.60 & 0.2 \\
\hline
\end{tabular}

\subsection{Preparation of specimens}

The concrete cylinder specimens with $100 \mathrm{~mm}$ diameter and $200 \mathrm{~mm}$ height were prepared for determination chloride penetration and rapid chloride permeability test (RCPT). In the first part, the concrete specimens were cured in water up to 365 days before subjected to RCPT test. The procedures to prepare the sample was called as 2-day preparation. First, the specimens were removed two (2) days earlier from water curing before RCPT to be conducted. Then, the cylindrical specimens were cut into slice samples approximately 50 $\pm 1 \mathrm{~mm}$ in thickness using water-cooled diamond saw. After cutting, the samples were left in room temperature before coated with waterproofing coating at the side of samples. The second day of preparation began with the desiccation process to water-saturate the specimens using desiccator chamber for 24 hours. The RCPT was performed after the samples cured for 3, 7, 28, 56, 92, 182 and 365 days.

The second part, the specimens for chloride penetration evaluation were taken out from water curing after cured for 7 days. Before exposed to $3 \%$ sodium chloride $(\mathrm{NaCl})$ solution, the specimens were sealed at the top and bottom parts. In this research, waterproofing membrane type T-100 water-based PU bituminous was used to prevent the ingress of chloride ions at the both parts. After the coating membrane dried, the specimens instantly immersed in $3 \% \mathrm{NaCl}$ solution for chloride exposure. All the specimens were immersed for 
$3,7,28,56,92,182$ and 365 days. At each interval of immersion, the concrete specimens were taken out and tested its chloride penetration.

\subsection{Testing procedures}

In this study, the testing method to determine the chloride penetration can be divided into two (2) comprises of rapid chloride permeability test (RCPT) and colorimetric method $\left(\mathrm{AgNO}_{3}\right.$ sprayed method). Under chloride permeability of NPC, HPC, UHPC and a series of nano metaclayed-UHPC specimens, the RCPT was adopted. It is widely accepted that the ability of concrete to resist ingress of chloride ions can be resulted by performance the RCPT [7]. The RCPT was conducted for determining the resistance of concrete to chloride ions penetration. The chloride penetration was accelerated into the concrete specimens by applying the direct current of 60 voltage for 6 hours. Then, the concrete permeability levels were classified based on charge passed as specified in standard as tabulated in Table 2. The RCPT procedures were confirming to ASTM C1202-05 specifications [26]. This test was measuring the amount of electrical current that passes through a $50 \pm 1 \mathrm{~mm}$ thick slice of concrete samples. The chloride penetration was accelerated into the samples by applying the direct current of 60 - voltage for 6 hours. A sodium chloride solution $(\mathrm{NaCl})$ is applied to one side of the slice and a sodium hydroxide $(\mathrm{HCl})$ solution is applied to the other side. At the end of 6 hours, the samples were removed from the cells and the amounts of coulombs passed through the samples were recorded.

Table 2. Chloride permeability classes based on charge passed [26]

\begin{tabular}{cc}
\hline Charge Passed, Coulombs & Permeability Classes \\
\hline$>4000$ & High \\
$2000-4000$ & Moderate \\
$1000-2000$ & Low \\
$100-1000$ & Very Low \\
$<100$ & Negligible \\
\hline
\end{tabular}

On the other hand, the chloride penetration depth profile of NPC, HPC, UHPC and a series of nano metaclayed-UHPC samples were determined by chemical method namely silver nitrate colorimetric method. Before the test was carried out, the concrete samples were taken out from $3 \% \mathrm{NaCl}$ solution after immersed for 3, 7, 28, 56, 92, 182 and 365 days. The exposed samples were left in room temperature to allow it dried for a while. Afterwards, the samples were prepared by splitting the samples axially into two (2) sections using compression machine. Then, the freshly fractured surfaces split (cross-section) of samples was sprayed with $0.1 \mathrm{M}$ silver nitrate $\left(\mathrm{AgNO}_{3}\right)$ solution and left in temperature room until sprayed solution was dried. Subsequently after the sprayed surface was completely dried, white silver chloride precipitation on the surface was visible. While, the area free of chloride ion remains natural or brown in colour. In order to measure the profile depth of the chloride precipitation, the line along the surface that contain chloride was drawn. Then, the penetration depth of the chloride penetrability was measured using digital caliper.

\section{Results and discussions}

\subsection{Rapid chloride permeability test (RCPT)}

The results of RCPT is presented in Fig. 1. The figure demonstrates the charge passed through the concrete decreases as the age prolonged for all concretes. The chloride 
permeability indication can be classified based on the charge passed into concrete as in accordance with ASTM C1202 specifications. At early age, it was revealed that the chloride permeability for NPC concrete can be identified as high level of permeability $(>40000$ Coulombs) as compare to those concrete mixes. After matured at 28-days, the chloride penetration in NPC concrete dropped up to 365-days. The Coulombs charge of the NPC concrete is reduced to 2676 Coulombs, indicating moderate chloride penetration resistance. The results also clear that the Coulombs charge through NPC is higher than HPC, UHPC and nano metaclayed-UHPC.

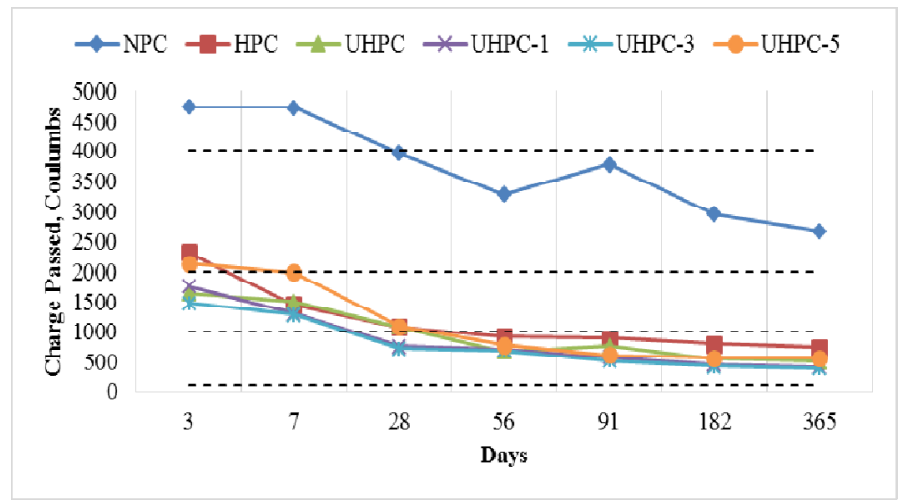

Fig. 1. Charge passed measured through NPC, HPC, plain UHPC and nano metaclayed-UHPC concretes

It was observed that, the presence of silica fume in HPC and nano metaclay in UHPC concretes contribute a benefit in terms of resisting chloride penetration. The incorporation of silica fume and nano metaclay in HPC and UHPC respectively, results in drastic reduction in the coulombs charge. The charge passed for HPC, UHPC and nano metaclay in UHPC concretes are reduced from low penetration to very low penetration of chloride from day-3 to day-56 respectively. In general, it is also revealed that small charge changes in Coulombs passed were observed for HPC, UHPC and a series of nano metaclayed-UHPC concretes after 28-days. This contrasting behavior occurs because of pozzolan-containing concrete increase more rapidly with increase in curing time than do those for the NPC concrete. On the other hand, it is also noted that higher $\mathrm{w} / \mathrm{c}$ ratio results in higher porosity, so that the total charge passed for NPC concrete is more connected than the pore structure in low w/c ratio concrete.

On the other hand, incorporation of nano metaclay in UHPC concretes had superior chloride resistance other than HPC concrete. The Coulombs passed of the UHPC and a series of nano metaclayed-UHPC slightly decreased after day-56. Among the UHPC concrete, the different is insignificant but the trend is still the same. It was expressed that incorporating 1, 3 and 5\% nano metaclay in UHPC can decrease RCPT results up to $76.11 \%, 72.67 \%$ and $73.59 \%$ respectively. Previous research also found that the volume of chloride that penetrated into the UHPC is extremely low [7]. These results are consistent with finding from other researchers, where they reported the UHPC have a discontinuously capillary porosity and thus exhibit significantly decreased the chloride permeability [27, 28]. In addition, among the series of nano metaclayed UHPC mixes, UHPC incorporating $1 \%$ nano metaclay (UHPC-1) has been proved the lowest chloride penetration compared with other nano metaclayed-UHPC mixes. Incorporating different percentage of nano metaclay in UHPC did not improve greatly the chloride resistance for all the nano metaclayed-UHPC from day-56 up until day-365. However, the reaction of $1 \%$ nano metaclay has the anticipated effect on the penetration characteristics of nano metaclayed- 
UHPC. It is believed that, the good chloride resistance of nano metaclay blended concretes is due to the refinement of the pore structure. Previous research also stated that nanoclay in UHPC is able to acts as an ultra-filler material and yields a significant reduction in the total charge passed [19].

\subsection{Chloride penetration depth profile}

The results highlighted the influence of silica fume in HPC and nano metaclay in producing UHPC concretes to chloride penetrability using colorimetric method. The chloride penetration depth profiles with respect to expose days in $3 \% \mathrm{NaCl}$ solution are graphically presented in Fig. 2 for all concrete mixes. Overall, it was found that the depth of chloride ion penetration in the concrete increased gradually with the time of exposure. Later, the chloride penetration depth decreased after expose for 365-days. As expected, the amount of chloride can be penetrated through NPC concrete recorded highest penetration depth compare to those other concrete series. It was noted that, the depth of the chloride penetration in NPC is significant because of the w/c ratio was high thus, results in higher pore size distribution. It is also clear that $\mathrm{w} / \mathrm{c}$ ratio is the governing factor for chloride ion penetration and is mostly dependent on concrete pore structure. Fig. 2 also shows the depth of chloride ion penetration was high for NPC and HPC from day-3 to day-91. Later, chloride depth in HPC was higher as compare to plain UHPC and UHPC with $1 \%$ and $3 \%$ of nano metaclay. In this stage, it is noticed that silica fume had a significant effect on reducing the chloride penetration due to the pozzolanic and micro-filler effect. This finding was also agreed by [29], who found that depth of chloride penetration in concrete made with silica fume was became zero at 91 days.

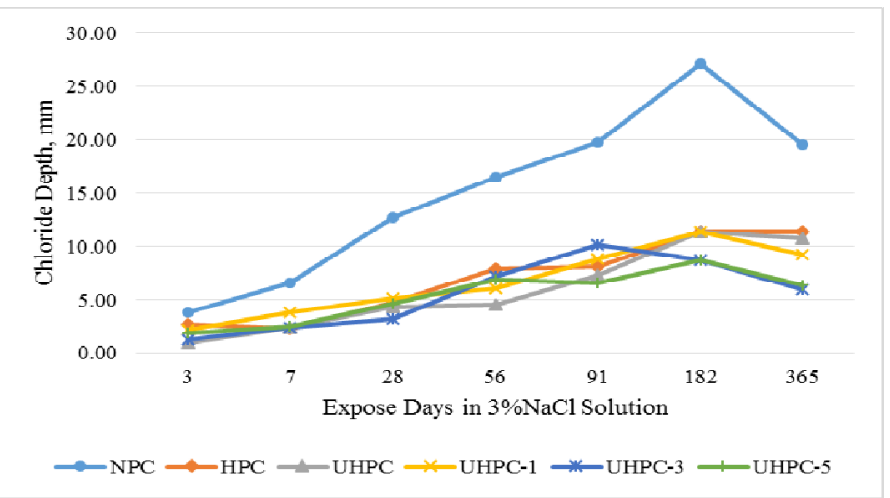

Fig. 2. Chloride penetration depth in NPC, HPC, UHPC and nano metaclayed-UHPC concretes

For UHPC concretes, it is revealed that as the nano metaclay level increases, there is a very marked reduction in the chloride resistance as compare to UHPC alone after 91-days. Due to its ultrafine size, nano metaclay particles fill the voids and reduce the capillary pores. At this stage, the hydration of UHPC concrete with nano metaclay have been also more advanced as a result of the additional hydration and pozzolanic reaction. The denser nano metaclayed UHPC would be less vulnerable to the ingress of the chloride in comparison to those at the age of 56-days. However, it has been observed that UHPC made with 3\% nano metaclay improved significantly the chloride resistance at 365-days of exposure. From the present results, it is clearly publicized that nano metaclay in UHPC produces finer pore structure due to formation of the pozzolanic reaction within the capillary pore space. The pore system will also become finer and more segmented with 
increase in exposure time due to continuing pozzolanic reaction. As well documented in previous studies, the used of nano-silica in concrete will delay and reduce the chloride resistance [13-16].

\subsection{Relationship on coulombs passed and chloride penetration depth}

In order to investigate the role of nano metaclay in enhancing the chloride resistance of the UHPC concrete, the relationship between Coulombs passed and chloride depth was performed. Fig. 3 displays the relationship between Coulombs passed and chloride penetration depth for series of nano metaclayed-UHPC concretes.

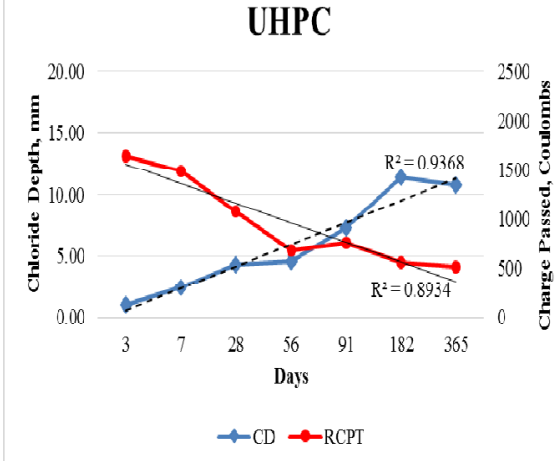

(a)

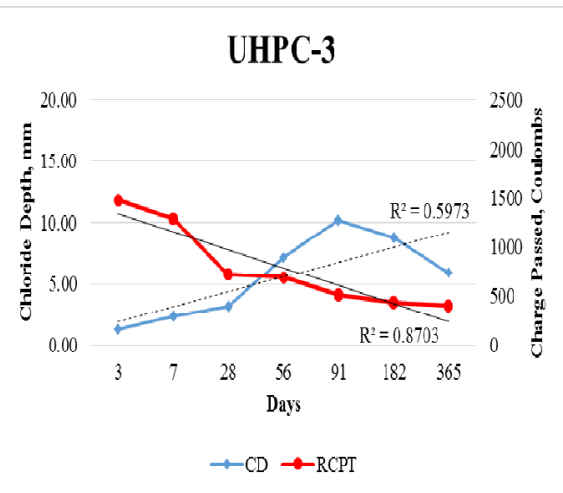

(c)

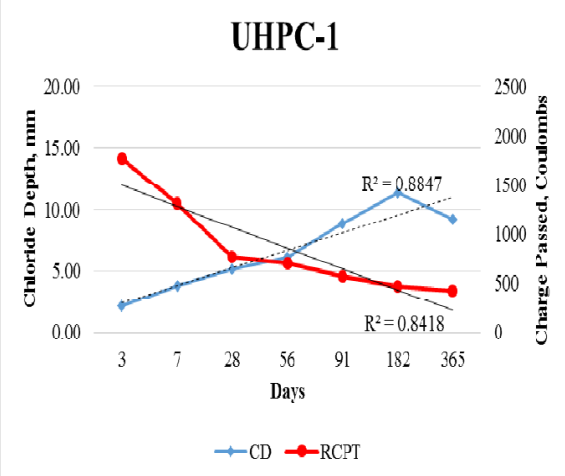

(b)

\section{UHPC-5}

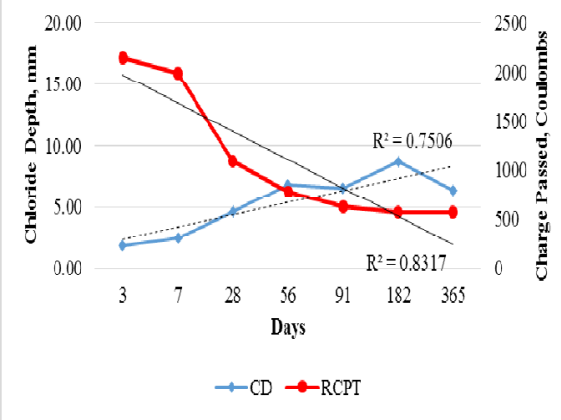

(d)

Fig. 3. Relationship between chloride depth and charge (Coulombs) passed for UHPC and nano metaclayed-UHPC

In general, the performance of chloride resistance of concrete can be improved by obtaining a discontinuous capillary pore structure caused by lowering w/c ratio or the presence of pozzolanic materials. In this case, the w/c ratio used for UHPC and nano metaclayed-UHPC concretes were constant at 0.2 . From the graph, it was revealed that the optimum performance of nano metaclay to contribute in the pozzolanic reaction was recorded at day56 for UHPC-1 and UHPC-5. However, the crossing line for UHPC was plotted just after 56-days and UHPC-3 was obtained nearly 56-days. This can give visual evidence that the degree of hydration in UHPC incorporating nano metaclay appeared considerably at day56. Therefore, the filler effect and the pore volume refinement occurred at 56-days of age. 
In order to verify the real influence of nano metaclay at 56-days, the relationship graph between chloride penetration and charge passed was performed as shown in Fig. 4. It is found that the different percentage of nano metaclay in UHPC has a strong linear with the chloride depth penetration. This mean $77.07 \%$ variation in chloride penetration depth profile has been explained by variation in the different levels of nano metaclay replacement and another $22.93 \%$ variation can be explained by other factors such as $\mathrm{w} / \mathrm{c}$ ratio or age of curing. Meanwhile, the relationship between RCPT charge passed and various replacement levels of nano metaclay was not very strong [R-square $=0.6559]$. This means that only $65.59 \%$ variation in charge passed has been explained by variation in the percentage of nano metaclay and the other of $34.41 \%$ can be explained by other factors. The graph indicates the optimum performance of chloride resistance for UHPC incorporating $1 \%$ nano metaclay was achieved on the day-56. Previous finding also found that the addition of metakaolin to concrete has a significant effect on to Coulombs passed and chloride penetration depth at early age [30]. Another researcher was also discovered that the use of nano metakaolin in concrete has reduced the capillary in concrete at 28 days of hydration [31].

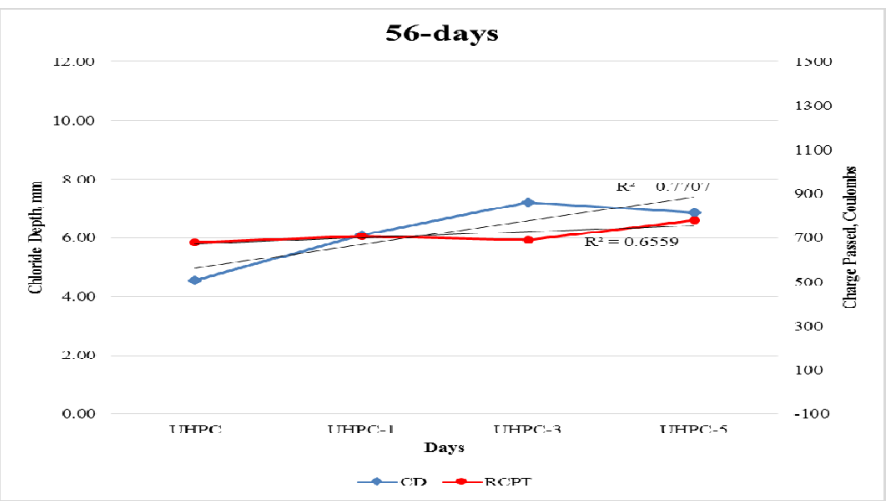

Fig. 4. Relationship on chloride depth and charge passed for UHPC and nano metaclayed-UHPC on the day-56

\section{Conclusions}

Based on the experimental results of this research, the following conclusions can be drawn:

i) It is deduced that the presence of silica fume and nano metaclay in concrete is effective in reducing Coulombs passed at 56-days up to 365 -days. Incorporating nano metaclay in UHPC has a significant effect on the chloride permeability. From the results, $1 \%$ nano metaclay is found to be the optimum amount to be used in UHPC production. At this replacement level, the Coulombs passed value significantly reduced up to $76.11 \%$.

ii) The depth of chloride ion penetration for nano metaclayed-UHPC concretes are much lesser than NPC, HPC and UHPC concretes. The capillary chloride penetration gradually decreased towards to 182-days. Contradicted to the results obtained from RCPT, it was found that inclusion of $3 \%$ nano metaclay significanty reduced the chloride penetration depth.

iii) The optimum chloride resistance behavior of nano metaclay in UHPC was achieved on the day-56. At this stage, it is noted that the high degree of the hydration process of nano metaclay was developed. It was concluded that the UHPC developed incorporating nano metaclay has significant potential on chloride resistance. 


\section{References}

[1] O. Kayali and B. Zhu, Corrosion performance of medium-strength and silica fume high-strength reinforced concrete in a chloride solution, Cement and Concrete Composition, 27, 117-124 (2005)

[2] M. Maes and N. De Belie, Resistance of concrete and mortar against combined attack of chloride and sodium sulphate, Cement and Concrete Composition, 53, 59-72 (2014)

[3] C. Andrade, R. d'Andrea and N. Rebolledo, Chloride ion penetration in concrete: The reaction factor in the electrical resistivity model, Cement and Concrete Composition, 47, 41-46 (2013)

[4] Federal Highway Administration, Building more durable bridges, FOCUS Federal Highway Administration, Washington DC, United States, (2001)

[5] BRE Centre for Concrete Construction, Guide to the maintenance, repair and monitoring of reinforced concrete structures, DME Report 4, Watford, United Kingdom, (2001)

[6] B.E. Graybeal and J.L. Hartmann, Strength and durability of ultra-high performance concrete, 3rd International Symposium on High Performance Concrete, PCI, Orlando, (2003)

Florida.

[7] B.E. Graybeal and J. Tanesi, Durability of an ultrahigh-performance concrete, J. of Materials in Civil Engineering, 19(10), 848-854 (2007)

[8] P.R. Prem, B.H. Bharatkumar and N.R. Iyer, Mechanical properties of ultra high performance concrete, Int. Scholarly and Scientific Research and Innovation, 6(8), 676-685 (2012)

[9] R. Yu, P. Spiesz and H.J.H. Brouwers, Mix design and properties assessment of ultrahigh performance fibre reinforced concrete (UHPFRC), Cement and Concrete Research, 56, 29-39 (2014)

[10]A.S. El-Dieb, Mechanical, durability and microstructural characteristics of ultrahighstrength self-compacting concrete incorporating steel fibres, Material and Design, 30, 4286-4292 (2009)

[11]B.A. Tayeh, B.H. Abu Bakar, M.A. Megat Johari and Y.L. Voo, Mechanical and permeability properties of the interface between normal concrete substrate and ultrahigh performance fibre concrete overlay, Construction and Building Materials, 36, 538-548 (2012)

[12]M. Schmidt, E. Fehling, C. Geisenhanslüke, Ultra high performance concrete (UHPC), structural material and engineering series, Proc. Symposium on Ultra High Performance Concrete, 3, Kassel, Germany, (2004)

[13]E. Ghafari, H. Costa, E. Júlio, A. Portugal and L. Durães, The effect of nanosilica addition on flowability, strength and transport properties of ultra-high performance concrete, Materials and Design, 59, 1-9 (2014)

[14]N. Randl, T. Steiner, S. Ofner, E. Baumgartner and T. Mészöly, Development of UHPC mixtures from an ecological point view, Construction and Building Materials, 67, 373-378 (2014)

[15]R. Yu, P. Spiesz and H.J.H. Brouwers, Effect of nano-silica on the hydration and microstructure development of ultra-high performance concrete (UHPC) with a low binder amount, Construction and Building Materials, 65, 140-150 (2014)

[16]M. Alkaysi, S. El-Tawil, Z. Liu and W. Hansen, Effects of silica powder and cement type on durability of ultra high performance concrete (UHPC), Cement and Concrete Composites,66(10),47-56 (2016)

[17]A.N. Mohammed, M.A.M. Johari, A.M. Zeyad, B.A. Tayeh and M.O. Yusuf, Improving the engineering and fluid transport properties of ultra-high strength concrete 
utilizing ultrafine palm oil fuel ash, J. of Advanced Concrete Technology, 12, 127-137 (2014)

[18] M.J.M. Faizal, M.S. Hamidah and M.S. Muhd Norhasri, Strength and chloride content of nanoclayed ultra-high performance concrete, Structures, Materials and Construction Engineering, DAKAM Publishing (2014)

[19] M.J. Mohd Faizal, M.S. Hamidah, M.S. Muhd Norhasri, I. Noorli and M.P. Mohamad Ezad Hafez, Chloride permeability of nanoclayed ultra-high performance concrete, Proc. of Int. Civil and Infrastructure Engineering Conf., Springer Singapore, 613-625 (2015)

[20] M.J. Mohd Faizal, M.S. Hamidah, M.S. Muhd Norhasri and I. Noorli, Effect of clay as a nanomaterial on corrosion potential of steel reinforcement embedded in ultra-high performance concrete, Proc. of Int. Civil and Infrastructure Engineering Conf., Springer Singapore, 679-689 (2016).

[21]C. Shi, Effect of mixing proportion of concrete on its electrical conductivity and the rapid chloride permeability test (ASTM C1202 or ASSHTO T227) results, Cement and Concrete Research, 34(3), 537-545 (2004)

[22] M.S. Ahmed, O. Kayali and W. Anderson, Evaluation of binary and ternary blends of pozzolanic materials using the rapid chloride permeability test, J. of Materials in Civil Engineering, 21(9), 446-453 (2009)

[23]K. Krishnakumar, S. Bhaskar and K. Parthlbah, Evaluation of chloride in OPC concrete by silver nitrate solution spray method, Int. J. of Chemical Technology Research, 6(5), 2676-2882 (2004)

[24]M.T. Bassuoni, M.L. Nehdi, and T.R. Greenough, Enhancing the reliability of evaluating chloride ingress in concrete using ASTM C1202 rapid chloride penetrability test, J. of ASTM International, 3(3), 1-13 (2006)

[25]M.R. Nooken and R.D. Hooton, Dependence of rate of absorption on degree of saturation of concrete, Journal of Cememt, Concrete and Aggregates (CCA), 24(1), 2024 (2002)

[26] ASTM C1202-07, Standard test method for the electrical indication of concrete's ability to resist chloride ion penetration, American Society of Testing and Materials, Pennsylvania, (2007)

[27]C. Vernet, UHPC microstructure and related durability performance laboratory assessment and field experience examples, Proc. of the Int. Symposium on High Performance Concrete, Orlando, (2003)

[28] O. Bonneau, C. Vernet, M. Moranville and P.C. Aïtchin, Characterization of the granular packing and percolation threshold of reactive powder concrete, Cement and Concrete Research, 26(11), 1639-1648 (2000)

[29] S. Ahmad, Development on test methods for assessment of concrete durability for use in performance-based specification, Master Thesis, University of Toronto, Canada, (2010)

[30]G. Dhinakaran, S. Thilgavathi and J. Venkataramana, Compressive strength and chloride resistance of metakaolin concrete, KSCE J. of Civil Engineering, 16(7), 12091217 (2012)

[31]H. Shouky, M.F. Kokkata, S.A. Abo-El-Enein, M.S. Morsy, S.S. Shebl, Enhanced physical, mechanical and microstructural properties of lightweight vermiculite cement composites modified with nano metakaolin, Construction and Building Materials, 112, 276-283 (2016) 